\title{
SPIN-UP AND PHASE FLUCTUATIONS IN THE TIMING OF THE ACCRETING MILLISECOND PULSAR XTE J1807-294
}

\author{
A. Riggio, ${ }^{1}$ T. Di Salvo, ${ }^{2}$ L. Burderi, ${ }^{1}$ M. T. Menna, ${ }^{3}$ A. Papitto, ${ }^{3}$ R. Iaria, ${ }^{2}$ and G. Lavagetto ${ }^{2}$ \\ Received 2007 June 28; accepted 2008 January 15
}

\begin{abstract}
We have performed a timing analysis of the 2003 outburst of the accreting X-ray millisecond pulsar XTE J1807-294 as observed by the Rossi X-Ray Timing Explorer. Using recently refined orbital parameters, we report for the first time a precise estimate of the spin frequency and of the spin frequency derivative. The phase delays of the pulse profile show a strong erratic behavior superposed on what appears to be a global spin-up trend. The erratic behavior of the pulse phases is strongly related to rapid variations of the light curve, making it very difficult to fit these phase delays with a simple formula. As in previous cases, we therefore separately analyze the phase delays of the first harmonic and of the second harmonic of the spin frequency, finding that the phases of the second harmonic are far less affected by the erratic behavior. Under the hypothesis that the second-harmonic pulse phase delays are a good tracer of the spin frequency evolution, we give for the first time an estimate of the spin frequency derivative for this source. XTE J1807-294 shows a clear spin-up of $\dot{\nu}=2.5(7) \times 10^{-14} \mathrm{~Hz} \mathrm{~s}^{-1}(1 \sigma$ confidence level). The majority of the uncertainty in the value of the spin-up rate is due to the uncertainties in the source position on the sky. We discuss the effect of this systematic error on the spin frequency and its derivative.
\end{abstract}

Subject headings: pulsars: general — pulsars: individual (XTE J1807-294) — stars: magnetic fields — stars: neutron - X-rays: binaries

Online material: color figure

\section{INTRODUCTION}

Binary systems in which one of the two stars is a neutron star (NS) are among the most powerful X-ray sources of our Galaxy. The emission of X-rays is due to the transfer of matter from the companion star and accretion onto the NS, and to the release of the immense gravitational energy during the infall or in the impact with the NS surface. A subcategory of such systems is referred to as low-mass X-ray binaries (LMXBs), which are characterized by low NS surface magnetic fields $\left(<10^{9} \mathrm{G}\right)$ and by the low mass $\left(<1 M_{\odot}\right)$ of the companion star. In the so-called recycling scenario (see Bhattacharya \& van den Heuvel 1991 for a review), the millisecond radio pulsars are seen as the last evolutionary step of LMXBs, where the torques due to the accretion of matter and angular momentum, coupled with the relatively weak magnetic fields, are able to spin up the NS to periods on the order of a millisecond. When the accretion phase terminates and the companion star stops transferring matter, the NS can switch on as a millisecond radio pulsar, although no example has been reported yet.

The recycling scenario received long-awaited confirmation only in 1998, with the discovery of the first millisecond X-ray pulsar in a transient LMXB: the first LMXB observed to show coherent pulsations, at a frequency of $\sim 400 \mathrm{~Hz}$, was SAX J1808.4-3658 (Wijnands \& van der Klis 1998), in which the NS is orbiting its companion star with a period of $\sim 2.5 \mathrm{hr}$ (Chakrabarty \& Morgan 1998). Why millisecond X-ray pulsars were so elusive is an argument still debated in the literature. A possible reason is the relatively low magnetic fields of these sources, which therefore

\footnotetext{
1 Dipartimento di Fisica, Università degli Studi di Cagliari, Cittadella Universitaria, I-09042 Monserrato, Italy; riggio@dsf.unica.it.

2 Dipartimento di Scienze Fisiche e Astronomiche, Università di Palermo, I-90123 Palermo, Italy.

3 Osservatorio Astronomico di Roma, Sede di Monteporzio Catone, I-00040 Rome, Italy.
}

have a lessened capability to channel the accreting matter onto the polar caps, making the chance of seeing pulsed emission from an LMXB quite low (see, e.g., Vaughan et al. 1994), especially at high accretion rates. However, to date 10 LMXBs have been discovered to be accreting millisecond pulsars (see Wijnands 2006 for a review of the first six discovered; for the last four, see Kaaret et al. 2006; Krimm et al. 2007; Casella et al. 2008; Altamirano et al. 2008), and all of them are in transient systems. They spend most of their time in a quiescent state, with very low luminosities (on the order of $10^{31}-10^{32} \mathrm{ergs} \mathrm{s}^{-1}$ ), and rarely go into an X-ray outburst, with luminosities in the range $10^{36}-10^{37} \mathrm{ergs} \mathrm{s}^{-1}$. Although the recycling scenario seems to be confirmed by these discoveries, from timing analyses of accreting millisecond pulsars we now know that some of these sources exhibit spin-down while accreting (Galloway et al. 2002; Papitto et al. 2007). This means that it is of fundamental importance to study the details of the mechanisms regulating the exchange of angular momentum between the NS and the accreting matter, which are far from being understood, and, chiefly, the role of the magnetic field in this exchange. The main way to do this is through the study of the pulse phase shifts and their relations with other physical observable parameters of the NS.

The pulse phase shifts are frequently affected by intrinsic longterm variations, fluctuations, or both (by which we mean an erratic behavior of the phase delays, possibly caused by variations in the instantaneous accretion torques or movements of the accretion footprints on the NS surface; see Di Salvo et al. 2007 for a review). Examples of this complex behavior in accreting millisecond pulsars have already been reported in the literature. Burderi et al. (2006) analyzed the 2002 outburst of the accreting millisecond pulsar SAX J1808.4-3658 and found a jump of 0.2 in the pulse phases of the first harmonic that is not present in the secondharmonic phases, which show much more regular behavior. This change corresponds to a change in the slope in the exponential decay of the X-ray light curve (see also Hartman et al. 2008 for 
a discussion of the complex phase behavior in other outbursts of SAX J1808.4-3658). Papitto et al. (2007) found that the second harmonic of XTE J1814-338 follows the first harmonic, giving approximately the same spin frequency derivative. A clear model that can explain this behavior is still lacking, but this observational evidence seems to suggest that perhaps the second harmonic has a more fundamental physical meaning. For instance, it may be related to the emission of both polar caps, while the first harmonic may be dominated by the more intense but less stable polar cap. Another possible explanation comes from possible shape and/or size variations of the accretion footprints related to variations in the accretion rate. Romanova et al. (2003) found such a behavior in their numerical simulations.

In this paper, we report the results of a timing analysis performed on XTE J1807-294, making use of an improved orbital solution (Riggio et al. 2007). As in the cases mentioned above, XTE J1807-294 shows erratic fluctuations in the phase delays of the first harmonic and a much more regular behavior of the phase delays derived from the second harmonic. Under the hypothesis that the second-harmonic pulse phase delays are a good tracer of the spin frequency evolution, we can derive a spin-up rate of $2.5(7) \times 10^{-14} \mathrm{~Hz} \mathrm{~s}^{-1}(1 \sigma$ confidence level $)$.

\section{OBSERVATION AND DATA ANALYSIS}

The millisecond X-ray pulsar XTE J1807-294 was discovered by the Rossi X-Ray Timing Explorer (RXTE) on 2003 February 21 (Markwardt et al. 2003c). The source was observed with the Proportional Counter Array (PCA; 2-60 keV energy range) and the High Energy X-Ray Timing Experiment (HEXTE; 20$200 \mathrm{keV}$ ) on board $R X T E$ (Jahoda et al. 1996) during a long X-ray outburst that lasted from 2003 February 28 to June 22. XTE J1807-294 was also observed with other satellites such as XMMNewton (Campana et al. 2003; Kirsch et al. 2004; Falanga et al. 2005), Chandra (Markwardt et al. 2003b), and INTEGRAL (Campana et al. 2003). No optical or radio counterpart has been reported to date. Linares et al. (2005) reported the presence of twin kilohertz quasi-periodic oscillations from an analysis of the RXTE observations.

Here we analyze all the archival $R X T E$ observations of this source. In particular, we employ high time resolution data from the PCA. We use data collected in "GoodXenon" packing mode for the timing analysis, which permits maximum time and energy resolution (respectively, $1 \mu$ s and 256 energy channels). In order to improve the signal-to-noise ratio $(\mathrm{S} / \mathrm{N})$, we select photon events from the top layers of the proportional counter units (PCUs) in the energy range 3-13 keV (Galloway et al. 2002). We have verified that this is the range where we indeed have the highest $\mathrm{S} / \mathrm{N}$. In fact, using the entire energy range, the pulsations at days 104 and 106 after the beginning of the outburst are much less statistically significant.

Using the faxbary ${ }^{4}$ tool (DE405 solar system ephemeris), we corrected the photon arrival times for the motion of the Earthspacecraft system and referred them to Barycentric Dynamical Times at the solar system barycenter. We use the source position reported by Markwardt et al. (2003b) from a Chandra observation during the same outburst.

To obtain the X-ray light curve during the outburst, we used the PCA data collected in Standard-2 mode (256 energy channels and $16 \mathrm{~s}$ binned data) and corrected for the background using the faintbackground model suitable for the source's count rate (see Jahoda

\footnotetext{
4 The faxbary tool is part of the HEAsoft software package. It can be found at http://heasarc.nasa.gov/docs/software/lheasoft.
}

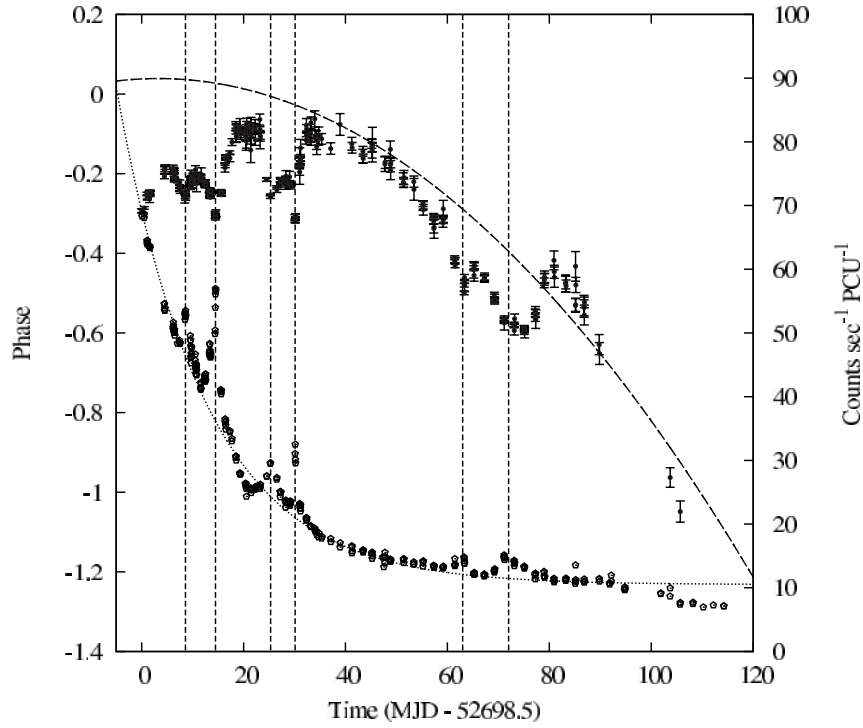

FIG. 1.- Light curve of XTE J1807-294 during the 2003 outburst ( pentagons) and phase delays of the first harmonic as a function of time (dots). The dashed vertical lines indicate the times of six clearly visible flares of the X-ray flux, superposed on a global exponential decay. The dotted curve represents the exponential fit of the light curve, obtained after having previously excluded the six flares from the data. The dashed curve represents the parabolic best fit obtained fitting the second-harmonic phase delays and considering the nominal source position. Strong fluctuations of the phase delays are apparent and are strongly anticorrelated with the flares present in the X-ray light curve.

et al. 2006). No energy selection was applied in this case, since we are interested in a good tracer of the bolometric luminosity. We also did not apply any correction for dead time, since the maximum count rate was quite low $\left(<100\right.$ counts $\mathrm{s}^{-1}$ per PCU, background included); in fact, the mean time between two events is at least 2 orders of magnitude higher than the expected dead time $(10 \mu \mathrm{s})$ for this count rate (Jahoda et al. 2006). We selected all the data using both internal Good Time Intervals and applying criteria regarding pointing offset, South Atlantic Anomaly (SAA) passage, electronic contamination, and Sun offset. ${ }^{5}$

The resulting light curve is shown in Figure 1 (pentagons). The flux exhibits an exponential decay, with six evident flares superposed. To derive the characteristic time of the decay, we fitted the light curve with an exponential law. In order to remove the time intervals affected by X-ray flares, we excluded from the fit all the points whose flux was greater than the best-fit exponential model by at least $15 \%$. This choice of threshold is arbitrary, but a different choice, such as $10 \%$ or $20 \%$, includes or excludes very few other points. We repeated the exponential fit on the flare-subtracted light curve. In this last fit, the $\chi^{2}$ was 23,096 for 214 degrees of freedom (dof), which is extremely high. Such a large $\chi^{2}$ is due to deviations of the X-ray light curve from pure exponential decay (see, e.g., all the points after 100 days from the beginning of the outburst).

Although these deviations may be very small intrinsically, they can be large in comparison with the statistical error on a single point. However, in order to obtain a reliable estimate of the parameters of the fit, and in particular a reliable estimate of the errors, we need to obtain a reduced $\chi^{2}$ of order unity. Therefore, we multiplied the errors on each point by a factor of 10 . In this way we obtain a characteristic decay time of $\tau=17.50(25)$ days.

5 According to the prescription given at http://heasarc.gsfc.nasa.gov/docs/ xte/abc/screening.html, we adopted as selection criteria the following: time since SAA greater than 30 minutes, elevation angle with respect to Earth greater than $10^{\circ}$, electron contamination lower than 0.1 , and pointing offset lower than $0.25^{\circ}$. 
TABLE 1

Orbital and Spin Parameters for XTE J1807-294

\begin{tabular}{|c|c|}
\hline Parameter & Value \\
\hline R.A. $(\mathrm{J} 2000) \ldots \ldots \ldots . .$. & $180659.8^{\mathrm{a}}$ \\
\hline 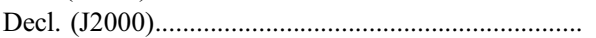 & $-292430^{\mathrm{a}}$ \\
\hline 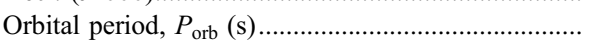 & $2404.41665(40)^{\mathrm{b}}$ \\
\hline Projected semimajor axis, $a_{x} \sin i(1 \mathrm{t}-\mathrm{ms}) \ldots \ldots \ldots \ldots \ldots \ldots \ldots$ & $4.819(4)^{\mathrm{b}}$ \\
\hline Ascending node passage, $T_{*}(\mathrm{MJD})$ & $52,720.675603(6)^{\mathrm{b}}$ \\
\hline Eccentricity, $e$ & $<0.0036^{\mathrm{b}}$ \\
\hline 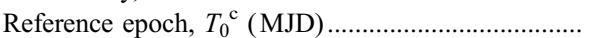 & $52,698.5$ \\
\hline \multicolumn{2}{|l|}{ Parabolic fit: } \\
\hline 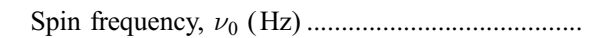 & $190.62350702(4)$ \\
\hline Spin frequency derivative, $\dot{\nu}\left(\mathrm{Hz} \mathrm{s}^{-1}\right) \ldots \ldots \ldots \ldots \ldots \ldots \ldots \ldots \ldots \ldots$ & $2.5(7) \times 10^{-14}$ \\
\hline \multicolumn{2}{|l|}{ Exponential fit: } \\
\hline 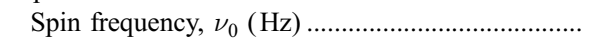 & $190.62350694(5)$ \\
\hline Spin frequency derivative, $\dot{\nu}_{0}\left(\mathrm{~Hz} \mathrm{~s}^{-1}\right) \ldots \ldots \ldots \ldots \ldots$ & $1.25(33) \times 10^{-13}$ \\
\hline \multicolumn{2}{|c|}{$\begin{array}{l}\text { NotEs.-Errors on orbital parameters are intended to be at the } 1 \sigma \text { confidence } \\
\text { level (c.l.); upper limits are given at } 95 \% \text { c.l. Best-fit spin parameters are derived } \\
\text { under both the hypothesis of a constant spin-up and of a flux-dependent spin-up, } \\
\text { and the uncertainties include systematics due to the uncertainties in the source } \\
\text { position (see text). Units of right ascension are hours, minutes, and seconds, and } \\
\text { units of declination are degrees, arcminutes, and arcseconds. } \\
\text { a Markwardt et al. } 2003 \mathrm{~b} \text {. } \\
\text { b Riggio et al. } 2007 \text {. } \\
\text { c This is the epoch to which the reported values of } \nu \text { and } \dot{\nu} \text { are referred. }\end{array}$} \\
\hline
\end{tabular}

It should be noted that a constant term must be added to the model to obtain a good description of the light curve, although background subtraction was performed. This residual amounts to $\sim 10.8(2)$ counts $\mathrm{s}^{-1}$ per PCU and may be due to a contaminating source in the PCA field of view. It is unlikely that it is due to quiescent emission, since the source was observed in quiescence by XMM-Newton and was not detected (Campana et al. 2005). In either case, this residual flux does not affect the inferred decay time of the light curve or any other results of this paper.

In order to minimize the time delays induced by the orbital motion, we correct the photon arrival times according to the formula

$$
\begin{aligned}
t_{\mathrm{em}} \simeq t_{\mathrm{arr}}-A\left\{\sin \left[m\left(t_{\mathrm{arr}}\right)+\omega\right]\right. \\
\left.+\frac{e}{2} \sin \left[2 m\left(t_{\mathrm{arr}}\right)+\omega\right]-\frac{3 e}{2} \sin \omega\right\}
\end{aligned}
$$

(see, e.g., Deeter et al. 1981), where $t_{\mathrm{em}}$ is the photon emission time, $t_{\mathrm{arr}}$ is the photon arrival time, $A$ is the projected semimajor axis in light-seconds, $m\left(t_{\text {arr }}\right)=2 \pi\left(t_{\text {arr }}-T_{*}\right) / P_{\text {orb }}$ is the mean anomaly with $P_{\text {orb }}$ the orbital period and $T_{*}$ the time of ascending node passage, $\omega$ is the periastron angle, and $e$ is the eccentricity. In order to remove completely from the pulse phase delays any effect due to the orbital motion, it is of fundamental importance to correct the arrival times of the events with very precise orbital parameters. To accomplish this, we used the orbital solution recently published by Riggio et al. (2007), who, using the total outburst time available (about 120 days), obtained a solution that is about 2 orders of magnitude more precise than previously reported orbital solutions.

We divided the whole observation into time intervals of length approximately equal to the orbital period ${ }^{6}$ and epoch-folded each of these data intervals with respect to the spin period reported

\footnotetext{
6 This is to minimize possible residuals due to uncertainties in the orbital parameters, since we expect these residuals to be periodic at the orbital period of the system.
}

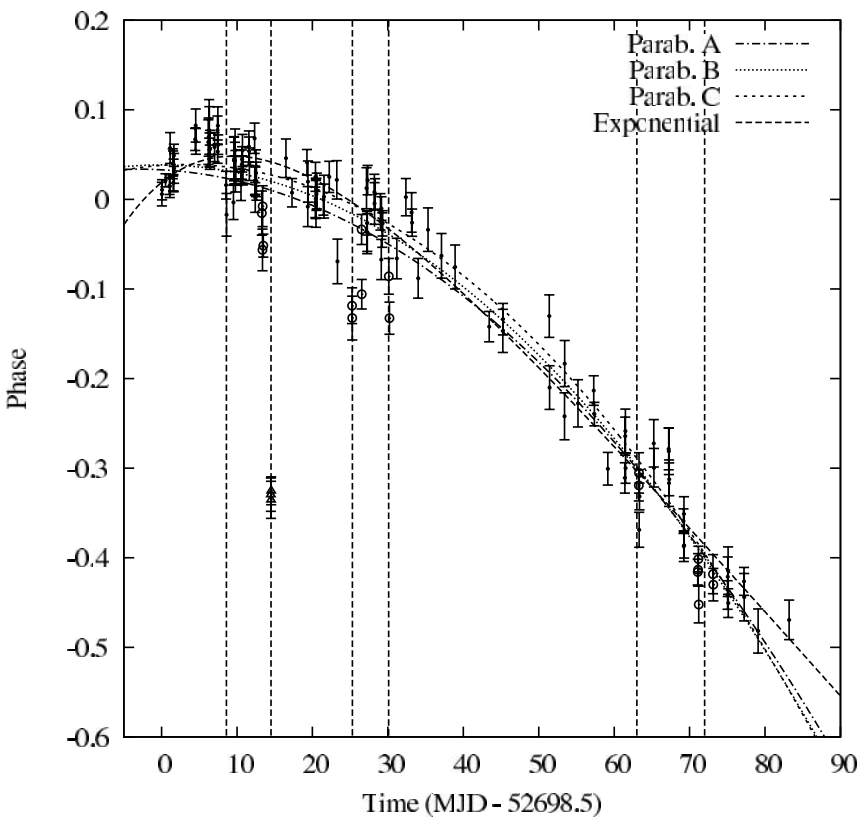

FIG. 2.-XTE J1807-294 second-harmonic pulse phase delays. The four curves represent the parabolic best fit for the nominal source position, respectively, using all the data points (case A), excluding the three points at MJD 52,713.0 (case B, where the points excluded are identified by triangles), and excluding all the data points for which the flux exceeds the best-fit exponential decay by more than $15 \%$ (case $\mathrm{C}$, where the points excluded are identified by circles), and the best fit obtained using an exponentially decreasing mass accretion rate (see text). The exponential fit was performed on the data subset corresponding to case C.

in Table 1. In this way, we were able to significantly detect the X-ray pulsations up to day 106 from the beginning of the outburst, making this the longest time span over which a timing analysis of an accreting millisecond pulsar has been performed.

The pulse phase delays are obtained by fitting each pulse profile with two sinusoidal components (with periods fixed to multiples of 1 and 0.5 of the spin period, respectively), since the second harmonic was significantly detected in the folded light curve. In Figures 1 and 2, we show the pulse phase delays of the first and second harmonics, respectively. We have plotted only the pulse phase delays corresponding to the folded light curves for which the statistical significance for the presence of X-ray pulsations is greater than $3 \sigma$. Moreover, we consider the second harmonic significantly detected (and plot its phases) only when the ratio between the best-fit amplitude of the second sinusoid and its error is greater than $3(A / \delta A>3)$. For each phase point, we propagated the errors on the orbital parameters with the formulae derived in Burderi et al. (2007). We note that the propagated errors in this case, for which the orbital parameters are known with great precision, come out to be much smaller than the statistical errors derived from the sinusoid fit.

As is evident from Figures 1 and 2, the phase delays of the first harmonic show a noisy behavior, with shifts of up to 0.3 in phase. This noise affecting the phase results is strongly anticorrelated with the source flux, as already noted for another source of this class (Papitto et al. 2007). On the other hand, the phase delays derived from the second harmonic are much more regular, a behavior that is similar to that exhibited by SAX J1808.4-3658 (Burderi et al. 2006). Although a few points (corresponding to rapid flares in the light curve) appear to be significantly below the general trend, the phase delays of the second harmonic clearly show a parabolic decrease, as is expected in the case of a spinning up of the NS. 


\section{TIMING RESULTS}

Since the phase delays of the second harmonic are much less noisy than the phases derived from the first harmonic, and assuming that the pulse phase delays derived from the second harmonic are a good tracer of the spin frequency evolution, we decided to fit the second harmonic in order to find information about the spin frequency behavior. To fit the phase delays, we start from the simplest assumption of a constant spin frequency derivative. We hence fit the second-harmonic phase delays with the model

$$
\phi(t)=\phi_{0}-\Delta \nu\left(t-T_{0}\right)-\frac{1}{2} \dot{\nu}\left(t-T_{0}\right)^{2}
$$

where $T_{0}$ is the date of the beginning of the observation, $\Delta \nu$ is a correction to the spin frequency, and $\dot{\nu}$ is the spin frequency derivative. Using all the data points, we obtain a spin frequency derivative $\dot{\nu}=2.05(28) \times 10^{-14} \mathrm{~Hz} \mathrm{~s}^{-1}$ with a quite large $\chi^{2 / \text { dof }}=$ $1560.57 / 142$. From a visual inspection of the phase residuals with respect to this model (see Fig. 2), we see that the largest contribution to the $\chi^{2}$ is from a group of three points at MJD 52,713.0 (about 14.5 days from the beginning of the outburst). These points (Fig. 2, triangles) correspond to the largest flare visible in the light curve and to a strong decrease of the phases of the first harmonic as well (cf. Fig. 1). We therefore believe that this is a phase shift induced by a rapid change of the X-ray flux similar to the phase shifts observed in the first harmonic. If we remove these three points from our data set ("case B") and perform the fit again, we obtain a frequency derivative $\dot{\nu}=2.26(15) \times 10^{-14} \mathrm{~Hz} \mathrm{~s}^{-1}$, perfectly compatible with the value previously found, demonstrating that the three points we have eliminated do not affect the spin frequency derivative obtained from the fit. In this case, of course, the statistical quality of the fit increases, giving $\chi^{2} /$ dof $=$ $452.4 / 139$. However, this $\chi^{2}$ is still unacceptable; again, the postfit residuals indicate that the major contribution to the $\chi^{2}$ statistic is due to all the points corresponding to the X-ray flares. We therefore decided to remove all the points (Fig. 2, circles) that fall in time intervals during which the flux is higher by $15 \%$ with respect to the exponential best-fit function derived above. In this way, a total of 21 points were excluded from the fit ("case C"). With this last data set we obtain a value for the spin frequency derivative $\dot{\nu}=2.46(15) \times 10^{-14} \mathrm{~Hz} \mathrm{~s}^{-1}$ (again compatible with the results obtained with the complete data set) and $\chi^{2} /$ dof $=257.6 / 121$. In this case, a value of $\nu_{0}=190.623507018(6) \mathrm{Hz}$ for the spin frequency at the beginning of the outburst is obtained.

We also tried to fit this (reduced) data set with a spin-up model that takes into account the decrease in the X-ray flux (supposed to trace the mass accretion rate) during the outburst (see Burderi et al. 2006 for a more detailed discussion). In principle, this correction should be important for this source given the particularly long duration of the outburst (about 120 days). Fitting the phase delays of the second harmonic with equation (1) of Burderi et al. (2006), in which we adopted an exponential decay time for the X-ray flux of 17.50 (25) days, as derived from the X-ray light curve, we obtain a significant improvement in the fit, with $\chi^{2} /$ dof $=225.5 /$ 121 ( $\Delta \chi^{2}=32$ for the same number of degrees of freedom). In this case, we obtain a spin frequency derivative at the beginning of the outburst of $\dot{\nu}_{0}=1.25(7) \times 10^{-13} \mathrm{~Hz} \mathrm{~s}^{-1}$, corresponding to a mass accretion rate at the beginning of the outburst of $\dot{M}_{0}=$ $4.03(23) \times 10^{-10} M_{\odot} \mathrm{yr}^{-1}$ and a best-fit spin frequency of $\nu_{0}=$ 190.623506939(7) Hz. In Figure 3 we report, for the last reduced data set, both parabolic and exponential best-fit models and the residuals from the exponential model (bottom).

Unfortunately, these results are affected by large systematic uncertainties due to the large uncertainty in the source coordinates

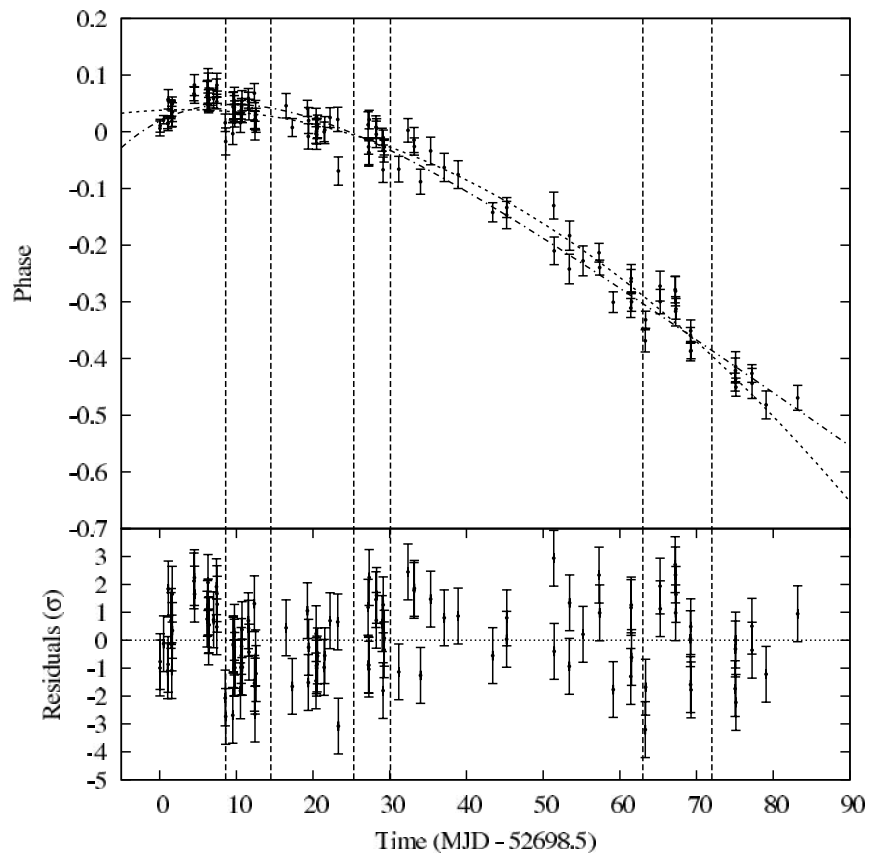

FIG. 3.-Second-harmonic pulse phase delays together with the parabolic and exponential best fit (top), and residuals in units of $\sigma$ with respect to the exponential best-fit model (bottom), considering only the subset of case C (see Fig. 2).

(about $0.4^{\prime \prime}$ [ 1 $\sigma$ confidence level $]$ from the Chandra observation), ${ }^{7}$ which we next discuss in detail.

Uncertainties in the phase delays caused by uncertainties in the estimate of the source position on the sky will produce a sinusoidal oscillation at Earth's orbital period. For observation times shorter than $1 \mathrm{yr}$, as is the case for most transient accreting millisecond pulsars, this can cause systematic errors in the determination of the NS spin period and its derivative, since a series expansion of a sinusoid contains both a linear and a quadratic term. In the case of XTE J1807-294, because of the low positional precision (Markwardt et al. 2003a) and the long time span over which the pulsations were visible (up to 106 days from the beginning of the outburst), we obtain, from the expression given in Burderi et al. (2007), the following systematic uncertainties on the spin frequency and the spin frequency derivative, respectively: $\sigma_{\nu, \text { pos }} \sim 4.1 \times 10^{-8} \mathrm{~Hz}$ and $\sigma_{\dot{\nu}, \text { pos }} \sim 0.8 \times 10^{-14} \mathrm{~Hz} \mathrm{~s}^{-1}$. Since this error is of the same order of magnitude as our best-fit estimate of $\dot{\nu}$, we need to evaluate these effects in a more careful manner.

Let us consider the expression for the phase delays induced by Earth's motion for small displacements, $\delta \lambda$ and $\delta \beta$, in the position of the source in ecliptic coordinates $\lambda$ and $\beta$ :

$$
\Delta \phi_{\text {pos }}(t)=\nu_{0} y\left[\delta \lambda \sin \left(M_{0}+\epsilon\right) \cos \beta-\delta \beta \cos \left(M_{0}+\epsilon\right) \sin \beta\right]
$$

(see, e.g., Lyne \& Graham-Smith 1990), where $y=r_{\mathrm{E}} / c$ is the distance of Earth from the solar system barycenter in light-seconds and $M_{0}=2 \pi\left(T_{0}-T_{\gamma}\right) / P_{\oplus}-\lambda$, with $T_{0}$ the beginning of the observation, $P_{\oplus}$ Earth's orbital period, $T_{\gamma}$ the time of passage

\footnotetext{
7 The Chandra observation of XTE J1807-294 in outburst was performed with the HRC-S instrument. As reported at http://asc.harvard.edu/cal/ASPECT/ celmon, the confidence levels are given at $68 \%\left(0.4^{\prime \prime}\right), 90 \%\left(0.6^{\prime \prime}\right)$, and $99 \%\left(0.8^{\prime \prime}\right)$ values.
} 

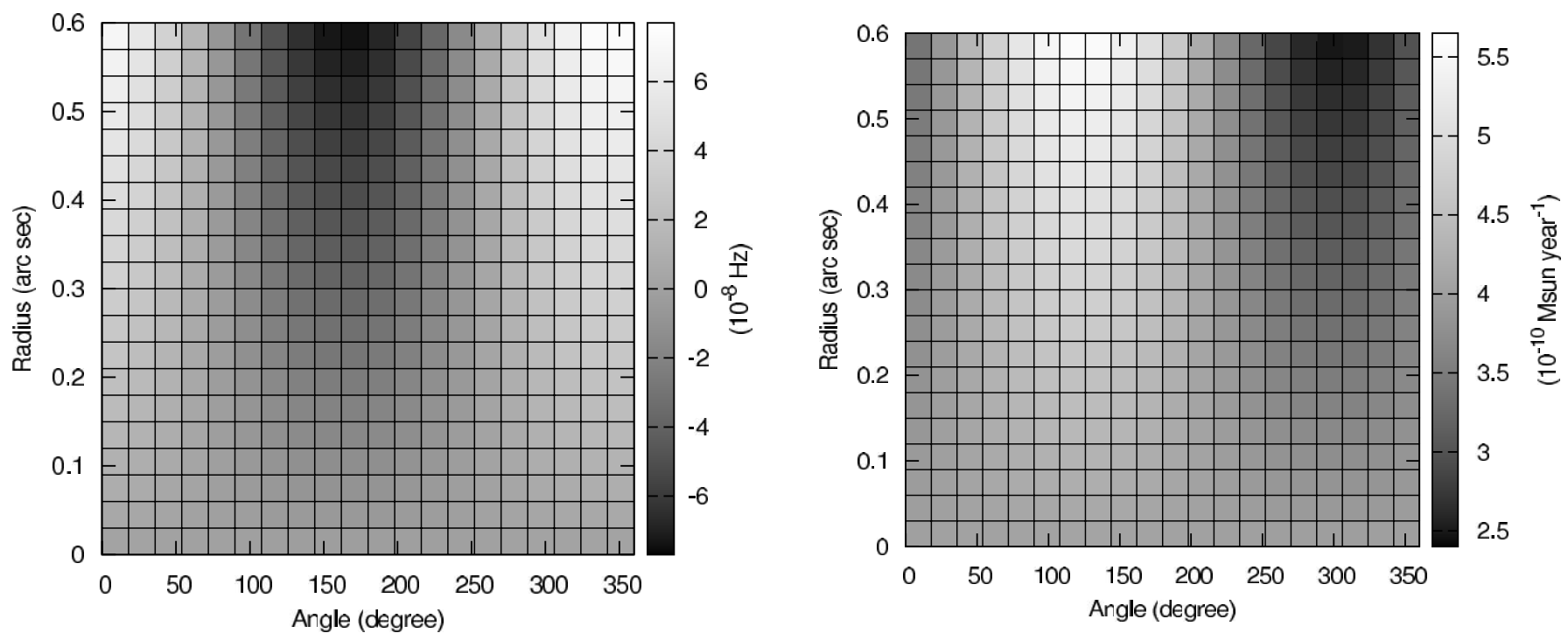

Fig. 4.-Diagrams of the best-fit values of $\Delta \nu$ (left $)$ and $\dot{M}$ (right) obtained by fitting the first-harmonic pulse phase delays with eq. (5), as functions of the parameters $\sigma_{\gamma}$ and $\theta^{*}$ (see text). [See the electronic edition of the Journal for a color version of this figure.]

through the vernal point, and $\epsilon=2 \pi\left(t-T_{0}\right) / P_{\oplus}$. As already calculated in Burderi et al. (2007) equation (3) can be rewritten as

$$
\Delta \phi_{\text {pos }}=\nu_{0} y \sigma_{\gamma} u \sin \left(M_{0}+\epsilon-\theta^{*}\right)
$$

where $\sigma_{\gamma}$ is the positional error circle, $\theta^{*}=\arctan [(\tan \beta) \delta \beta / \delta \lambda]$, and $u=\left[(\delta \lambda \cos \beta)^{2}+(\delta \beta \sin \beta)^{2}\right]^{1 / 2} / \sigma_{\gamma}$. We can safely impose $u=1$ as an upper limit.

In order to take into account the effects of an incorrect source position, we fitted the reduced data set (case $\mathrm{C}$ ) with a model that also takes into account the modulation caused by the incorrect source coordinates as given by equation (4):

$$
\phi(t)=\phi_{0}-\Delta \nu\left(t-T_{0}\right)-\frac{1}{2} \dot{\nu}\left(t-T_{0}\right)^{2}+\Delta \phi_{\text {pos }}(t) .
$$

We repeated the fit, changing $\sigma_{\gamma}$ and $\theta^{*}$ in such a manner as to cover the Chandra error box up to the $90 \%$ confidence level, that is, a sky region within an angular distance of $0.6^{\prime \prime}$ from the reported source position. The obtained values of the spin frequency and mass accretion rate for each possible position of the source within the Chandra error box are shown in Figure 4. The values of $\dot{\nu}$, at the $1 \sigma$ confidence level, lie in the interval $(1.8-3.2) \times 10^{-14} \mathrm{~Hz} \mathrm{~s}^{-1}$, while the best-fit value of the frequency derivative for the nominal source position is $2.46(15) \times 10^{-14} \mathrm{~Hz}$ $\mathrm{s}^{-1}$. It is evident that the effect of the poor knowledge of the source position is much larger than the statistical error on the parabolic fit. Still, the spin-up behavior of the source remains significant even considering the large uncertainties caused by the positional uncertainties.

A similar discussion is required for the spin frequency. The bestfit value, for the nominal position, is $\nu=190.623507018(4) \mathrm{Hz}$, while the variations of the linear term in the fit at different positions of the source inside the Chandra error box are in the range $\Delta \nu= \pm 4 \times 10^{-8} \mathrm{~Hz}$, an order of magnitude greater than the single fit statistical error. Finally, the reduced $\chi^{2}$ for these fits varies within the range $2.1-2.4$.

To summarize, using the pulse phase delays derived from the second harmonic, we have inferred the spin frequency derivative of XTE J1807-294. Under the hypothesis of a constant spin frequency derivative, we obtain a value $\dot{\nu}=2.46(15) \times 10^{-14} \mathrm{~Hz} \mathrm{~s}^{-1}$. Under the alternative hypothesis of an exponential decay of the accretion rate, we obtain a value for the spin frequency derivative at the beginning of the outburst of $\dot{\nu}_{0}=1.25(7) \times 10^{-13} \mathrm{~Hz} \mathrm{~s}^{-1}$. These results do not include the systematic errors induced by the poorly constrained source position. Taking into account the errors on the source position obtained above, for the constant and exponential decay models, respectively, the values are $2.5(7) \times$ $10^{-14} \mathrm{~Hz} \mathrm{~s}^{-1}$ and $1.25(33) \times 10^{-13} \mathrm{~Hz} \mathrm{~s}^{-1}$.

\section{DISCUSSION AND CONCLUSION}

We have analyzed a long $R X T E$ observation of the accreting millisecond pulsar XTE J1807-294 and reported the results of an accurate timing analysis on a time span of about 120 days, the longest outburst of an accreting millisecond pulsar for which a timing analysis has been performed to date. We find that the phase delays derived from the first harmonic show an erratic behavior around a global parabolic spin-up trend. This behavior is similar to that previously discovered in two accreting millisecond pulsars, SAX J1808.4-3658 (Burderi et al. 2006) and XTE J1814-338 (Papitto et al. 2007). In the case of the 2002 outburst of SAX J1808.4-3658, the phase delays of the first harmonic show a shift by about 0.2 in phase at day 14 from the beginning of the outburst, when the X-ray flux abruptly changed the slope of the exponential decay. On the other hand, the phase delays of the second harmonic in SAX J1808.4-3658 showed no sign of the phase shift in the first harmonic and could be fitted by a spinning up during the first part of the outburst plus a barely significant spin-down at the end. In the case of XTE J1814-338, the fluctuations in the phase delays are visible both in the first harmonic and in the second harmonic, superposed on a global parabolic spin-down trend. Papitto et al. (2007) showed that the postfit phase residuals are strongly anticorrelated with variations of the X-ray light curve. These fluctuations were interpreted as being due to movements of the accretion footprints (or accretion column) induced by variations of the $\mathrm{X}$-ray flux.

In the case of XTE J1807-294, the fluctuations in the phase delays mostly affect the first harmonic, which displays a trend that is very difficult to reproduce with a simple model. As in the case of XTE J1814-338, the postfit phase residuals are clearly anticorrelated with variations observed in the X-ray light curve; from Figure 1, we see that the phases decrease when the X-ray flux shows rapid increases. It is important to note that the anticorrelation 
visible between the postfit phase delays and the X-ray flux is independent of the spin-down or spin-up behavior of the source, since it is observed in XTE J1814-338, which shows spin-down, and in XTE J1807-294, which shows spin-up. The correlation between the phase delays and the X-ray flux affects the second harmonic only marginally. Indeed, there are a few points in the phase delays of the second harmonic that are significantly below the global trend observed in the phase delays, and they all correspond to flares in the X-ray light curve. Excluding these points marginally affects the values we obtain for the spin frequency and its derivative but produces a significant improvement in the $\chi^{2}$ of the fit.

We find that the phase delays of the second harmonic can be fitted by a parabolic spin-up model. We have also shown that the quality of the fit is much improved if a more physical model is used in which the spin-up rate decreases exponentially with time following the decrease of the X-ray flux (and hence of the inferred mass accretion rate). In fact, if the spin-up of the source is related to the mass accretion rate, then it should not be constant with time but, to first approximation, should decrease in proportion with the mass accretion rate onto the NS. For instance, assuming that the accretion of matter and angular momentum occurs at the corotation radius $R_{\mathrm{co}}$, the relation between the spin frequency derivative and the mass accretion rate is, from the conservation of angular momentum, $\dot{\nu}=\dot{M}\left(G M R_{\mathrm{co}}\right)^{1 / 2} / 2 \pi I$, where $G$ is the gravitational constant, $M$ is the NS mass, and $I$ is the NS moment of inertia; this gives a lower limit on the mass accretion rate, since the specific angular momentum at the corotation radius is the maximum that can be transferred to the NS. In the case of XTE J1807-294, the duration of the observed outburst is particularly long (about 120 days), and the effect of a global decrease of the mass accretion rate during the outburst should be particularly relevant for this source. Indeed, in this case the fit we obtain using an exponentially decreasing spin-up rate is significantly better than that using a constant spin-up rate.

From the fit of the phase delays of the second harmonic of XTE J1807-294 with the model discussed above, we find a mass accretion rate at the beginning of the outburst of $4(1) \times 10^{-10} M_{\odot}$ $\mathrm{yr}^{-1}{ }^{8}$ This mass accretion rate can be compared with the X-ray flux of the source at the beginning of the outburst, which was $2 \times 10^{-9} \mathrm{ergs} \mathrm{cm}^{-2} \mathrm{~s}^{-1}$ (Falanga et al. 2005), and from which we derive an X-ray luminosity of $4.7 \times 10^{36} \mathrm{ergs} \mathrm{s}^{-1}$ and a distance to the source of 4.4(6) kpc. Clearly, this is only a crude estimate of the distance on the basis of our timing results, and future independent estimates are needed in order to confirm or disprove our hypothesis.

This work was supported by the Ministero dell'Istruzione, dell'Università e della Ricerca (MIUR), national program PRIN2005, No. 2005024090004.

\footnotetext{
${ }^{8}$ For this estimate we adopted values of $I=10^{45} \mathrm{~g} \mathrm{~cm}^{2}, M=1.4 M_{\odot}$, and NS radius $R_{\mathrm{NS}}=10^{6} \mathrm{~cm}$.
}

Altamirano, D., Casella, P., Patruno, A., Wijnands, R., \& van der Klis, M. 2008, ApJ, 674, L45

Bhattacharya, D., \& van den Heuvel, E. P. J. 1991, Phys. Rep., 203, 1

Burderi, L., Di Salvo, T., Menna, M. T., Riggio, A., \& Papitto, A. 2006, ApJ, 653, L133

Burderi, L., et al. 2007, ApJ, 657, 961

Campana, S., Ferrari, N., Stella, L., \& Israel, G. L. 2005, A\&A, 434, L9

Campana, S., Ravasio, M., Israel, G. L., Mangano, V., \& Belloni, T. 2003, ApJ, 594, L39

Casella, P., Altamirano, D., Patruno, A., Wijnands, R., \& van der Klis, M. 2008, ApJ, 674, L41

Chakrabarty, D., \& Morgan, E. H. 1998, Nature, 394, 346

Deeter, J. E., Boynton, P. E., \& Pravdo, S. H. 1981, ApJ, 247, 1003

Di Salvo, T., Burderi, L., Riggio, A., Papitto, A., \& Menna, M. T. 2007, in AIP Conf. Proc. 924, The Multicolored Landscape of Compact Objects and Their Explosive Origins, ed. L. A. Antonelli et al. (Melville, NY: AIP), 613

Falanga, M., et al. 2005, A\&A, 436, 647

Galloway, D. K., Chakrabarty, D., Morgan, E. H., \& Remillard, R. A. 2002, ApJ, 576, L137

Hartman, J. M., et al. 2008, ApJ, 675, 1468

Jahoda, K., Markwardt, C. B., Radeva, Y., Rots, A. H., Stark, M. J., Swank, J. H., Strohmayer, T. E., \& Zhang, W. 2006, ApJS, 163, 401

Jahoda, K., Swank, J. H., Giles, A. B., Stark, M. J., Strohmayer, T., Zhang, W., \& Morgan, E. H. 1996, Proc. SPIE, 2808, 59

\section{EFERENCES}

Kaaret, P., Morgan, E. H., Vanderspek, R., \& Tomsick, J. A. 2006, ApJ, 638, 963 Kirsch, M. G. F., Mukerjee, K., Breitfellner, M. G., Djavidnia, S., Freyberg, M. J., Kendziorra, E., \& Smith, M. J. S. 2004, A\&A, 423, L9

Krimm, H. A., et al. 2007, ApJ, 668, L147

Linares, M., van der Klis, M., Altamirano, D., \& Markwardt, C. B. 2005, ApJ, 634,1250

Lyne, A. G., \& Graham-Smith, F. 1990, Pulsar Astronomy (Cambridge: Cambridge Univ. Press)

Markwardt, C. B., Juda, M., \& Swank, J. H. 2003a, ATel, No. 127

2003b, IAU Circ. 8095

Markwardt, C. B., Smith, E., \& Swank, J. H. 2003c, ATel, No. 122

Papitto, A., Di Salvo, T., Burderi, L., Menna, M. T., Lavagetto, G., \& Riggio, A. 2007, MNRAS, 375, 971

Riggio, A., Di Salvo, T., Burderi, L., Iaria, R., Papitto, A., Menna, M. T., \& Lavagetto, G. 2007, MNRAS, 382, 1751

Romanova, M. M., Ustyugova, G. V., Koldoba, A. V., Wick, J. V., \& Lovelace, R. V. E. 2003, ApJ, 595, 1009

Vaughan, B. A., et al. 1994, ApJ, 435, 362

Wijnands, R. 2006, in Trends in Pulsar Research, ed. J. A. Lowry (New York: Nova Sci.)

Wijnands, R., \& van der Klis, M. 1998, Nature, 394, 344 